\title{
The Necessity of Awareness of Early Symptoms of Placental Abruption Among Pregnant Japanese Women
}

\author{
Shunji Suzuki ${ }^{a, b}$, Hiroki Shinmura ${ }^{a}$
}

\begin{abstract}
Background: In 2012, the recommendation for immediate contact and visit to obstetric institutions by pregnant women was emphasized by The Japan Obstetric Compensation System for Cerebral Palsy (JOCSC). In this study, we examined whether or not the increased awareness has led to the improvement of perinatal outcomes of placental abruption managed at private clinics.
\end{abstract}

Methods: We reviewed the obstetric records of 38 singleton pregnant women complicated by placental abruption that developed at home, and were managed at private clinics from April 2008 through April 2016.

Results: The perinatal outcomes, specifically the rate of cases with $\geq$ 1 hour time interval between symptom onset and clinic visit, have not changed significantly after the intervention.

Conclusion: The provision of information regarding the early clinical symptoms associated with placental abruption in pregnant women has not been well documented in Japan.

Keywords: Placental abruption; Early symptoms; Provision of information; Japan

\section{Introduction}

The Japan Obstetric Compensation System for Cerebral Palsy (JOCSC) was launched in January 2009 to compensate for the economic burden of children with severe cerebral palsy (CP) associated with delivery and to conduct an analysis of the factors contributing to $\mathrm{CP}$, with the goal of improving the quality

\section{Manuscript accepted for publication July 18, 2016}

aDepartment of Obstetrics and Gynecology, Japanese Red Cross Katsushika Maternity Hospital, Tokyo, Japan

${ }^{b}$ Corresponding Author: Shunji Suzuki, Department of Obstetrics and Gynecology, Japanese Red Cross Katsushika Maternity Hospital, 5-11-12 Tateishi, Katsushika-ku, Tokyo 124-0012, Japan. Email: czg83542@mopera.ne.jp

doi: http://dx.doi.org/10.14740/jocmr2662w of health care of the Japanese. In the second annual report of JOCSC on May 2012 [1], a history of placental abruption was identified in $20(25.3 \%)$ of 79 cases of severe CP. Therefore, immediate contact and visit to obstetric institutions has been recommended for pregnant women with symptoms such as abdominal pains, genital bleeding and decreased fetal movements, because women take longer to visit medical institutions $[1,2]$. In addition, about half of all deliveries in Japan are managed at private clinics; patients with placental abruption managed at private clinics need to be transported to perinatal centers for prompt intervention [3]. Moreover, obstetricians were informed that the symptoms of placental abruption appear to be similar to those of preterm labor [1]. In this study, we examined whether or not awareness of the condition has led to the improvement of perinatal outcomes of placental abruption managed at private clinics.

\section{Methods}

The protocol for this study was approved by the Ethics Committee of the Japanese Red Cross Katsushika Maternity Hospital. Informed consent concerning analysis from a retrospective database was obtained from all subjects.

We reviewed the obstetric records of 38 singleton pregnant women complicated by placental abruption that developed at home, and were managed at private clinics from April 2008 through April 2016. Placental abruption was defined as separation of a normally implanted placenta, based on massive retro-placental bleeding after 24 weeks of gestation. All cases were delivered by cesarean section because of a non-reassuring fetal status and/or an immature cervix. There were no cases with prior placental abruption, preterm labor, or hypertensive disorders requiring ambulatory management. In this study, the 38 cases with placental abruption were divided into two groups of before $(n=20)$ and after $(n=18)$ the intervention. We examined the perinatal characteristics and outcomes, including maternal age, parity, gestational age at delivery, time interval between symptom onset and clinic visit, time interval between clinic visit and delivery, intrauterine fetal demise (IUFD), umbilical artery (UA) $\mathrm{pH}$ less than 7.0, postpartum hemorrhage (PPH) requiring hemotransfusion, and maternal disseminated intravascular coagulation (DIC).

Data were presented as numbers (\%). For the statistical 
Table 1. Perinatal Characteristics and Outcomes of 38 Patients Complicated by Placental Abruption That Developed at Home and Managed at Private Clinics

\begin{tabular}{llll}
\hline & Before the enlightenment & After the enlightenment & P-value \\
\hline Number of patients & 20 & 18 & $6(33 \%)$ \\
Maternal age $\geq 35$ years & $9(45 \%)$ & $15(83 \%)$ & 0.463 \\
Nulliparity & $12(60 \%)$ & $17(94 \%)$ & 0.113 \\
Gestational age $<37$ weeks & $18(90 \%)$ & $16(89 \%)$ & 0.612 \\
Neonatal birth weight $<2,500 \mathrm{~g}$ & $17(85 \%)$ & $16(78 \%)$ & 0.723 \\
Time interval from symptom onset to clinic visit $\geq 60$ min & $15(75 \%)$ & $0(0 \%)$ & 0.270 \\
Intrauterine fetal demise & $2(10 \%)$ & 18 & 0.189 \\
Live births & 18 & $8(44 \%)$ \\
Time interval from clinic visit to delivery $\geq 60$ min & $15(83 \%)$ & $3(17 \%)$ \\
Umbilical artery pH $<7$ & $6(33 \%)$ & $6(33 \%)$ \\
Total blood loss $\geq 2,000 \mathrm{~g}$ & $8(40 \%)$ & $9(50 \%)$ \\
Hemotransfusion & $11(55 \%)$ & $6(33 \%)$ \\
Disseminated intravascular coagulation & $9(50 \%)$ & 0.015 \\
\hline
\end{tabular}

analysis, the $\mathrm{X}^{2}$ test with Yates' correction was used for categorical variables. A P-value $<0.05$ was considered significant.

\section{Results}

Table 1 shows the perinatal characteristics and outcomes of the 38 patients complicated by placental abruption that developed at home and managed in private clinics. As shown in Table 1, the rate of cases with $\geq 1 \mathrm{~h}$ time interval between clinic visit and delivery decreased significantly after the intervention. There were no significant differences between the two groups in the other variables, such as perinatal outcomes and the rate of cases with $\geq 1 \mathrm{~h}$ time interval between symptom onset and clinic visit.

\section{Discussion}

In cases of severe placental abruption, a short time interval between decision and delivery has been observed to contribute to the substantially reduced neonatal morbidity and mortality [3, 4]. Although the decreased rate of cases with $\geq 1 \mathrm{~h}$ time interval between clinic visit and delivery did not contribute to the improvement of perinatal outcomes of placental abruption in this study, it suggests an increase in the awareness of placental abruption in the obstetricians working at clinics. On the other hand, the rate of cases with $\geq 1 \mathrm{~h}$ time interval between symptom onset and clinic visit has not changed measurably after the intervention. It may suggest that the provision of information regarding early clinical symptoms associated with placental abruption to pregnant women has not been well documented in Japan.

This study may be small, but it gave us a serious overview of the problem concerning the necessity of awareness of early symptoms of placental abruption for pregnant women in Japan.

\section{Conflicts of Interest}

The authors report no conflicts of interest.

\section{References}

1. Japan Council for Quality Health Care: The second Annual Report of the Japan Obstetric Compensation System for Cerebral Palsy (in Japanese). 2012. http://www. sanka-hp.jcqhc.or.jp/documents/prevention/report/pdf/ Saihatsu_Report_02_All.pdf(June 5, 2016).

2. Minakami H, Hiramatsu Y, Koresawa M, Fujii T, Hamada H, Iitsuka Y, Ikeda T, et al. Guidelines for obstetrical practice in Japan: Japan Society of Obstetrics and Gynecology (JSOG) and Japan Association of Obstetricians and Gynecologists (JAOG) 2011 edition. J Obstet Gynaecol Res. 2011;37(9):1174-1197.

3. Suzuki S. Clinical significance of adverse outcomes of placental abruption developing at home. J Obstet Gynaecol. 2015;35(4):433-434.

4. Kayani SI, Walkinshaw SA, Preston C. Pregnancy outcome in severe placental abruption. BJOG. 2003;110(7):679-683. 